\title{
Unicorn: Parallel adaptive finite element simulation of turbulent flow and fluid-structure interaction for deforming domains and complex geometry
}

\author{
Johan Hoffman ${ }^{\mathrm{a}}$, Johan Jansson ${ }^{\mathrm{a}}$, Rodrigo Vilela de Abreu ${ }^{\mathrm{a}}$, Niyazi Cem Degirmenci ${ }^{\mathrm{a}}$, Niclas Jansson ${ }^{\mathrm{a}, *}$, Kaspar Müller $^{\mathrm{a}}$, Murtazo \\ Nazarov $^{\mathrm{a}}$, Jeannette Hiromi Spühler ${ }^{\mathrm{a}}$ \\ ${ }^{a}$ Computational Technology Laboratory, CSC/NA, KTH, Royal Institute of Technology, SE-100 44 Stockholm, Sweden
}

\begin{abstract}
We present a framework for adaptive finite element computation of turbulent flow and fluid-structure interaction, with focus on general algorithms that allow for complex geometry and deforming domains. We give basic models and finite element discretization methods, adaptive algorithms and strategies for efficient parallel implementation. To illustrate the capabilities of the computational framework, we show a number of application examples from aerodynamics, aero-acoustics, biomedicine and geophysics. The computational tools are free to download open source as Unicorn, and as a high performance branch of the finite element problem solving environment DOLFIN, both part of the FEniCS project.
\end{abstract}

Keywords: Unicorn, DOLFIN, FEniCS, parallel adaptive finite element method, open source software, turbulent flow, fluid-structure interaction, complex geometry, deforming domain

\section{Introduction}

In this paper we give an overview of our work on finite element simulation of turbulent flow and fluid-structure interaction for complex geometry and deforming domains, in the form of a computational framework with focus on adaptive algorithms for parallel computer architectures. We present our open source implementation of the algorithms in the form of a high performance branch of the finite element problem solving environment DOLFIN ${ }^{1,2}$ and the unified continuum mechanics solver Unicorn ${ }^{3}$. DOLFIN and Unicorn are parts of the FEniCS project ${ }^{4}$, with the goal to automate the scientific software process by relying on general implementations and code generation, for robustness and to enable high speed of software development. To illustrate the capacity of the computational tools, we present snapshots from a number of application projects, together with parallel performance results.

We target a large family of problems of continuum mechanics, including incompressible and compressible flow, and fluidstructure interaction, described by (i) conservation of mass, (ii) balance of momentum, and (iii) conservation of energy, together with constitutive laws for fluids and solids. The basic laws (i)-(iii) take a generic form and can thus be handled by a general discretization strategy, with a common implementation. The constitutive laws, on the other hand, are specific for each problem, and are treated as data. The current implementation of

\footnotetext{
${ }^{*}$ Corresponding author

Email addresses: jhoffman@csc.kth.se (Johan Hoffman), jjan@csc.kth.se (Johan Jansson), rvda@csc.kth.se (Rodrigo Vilela de Abreu), ncde@csc.kth.se (Niyazi Cem Degirmenci), njansson@csc.kth.se (Niclas Jansson), kasparm@csc.kth.se (Kaspar Müller), murtazo@csc.kth.se (Murtazo Nazarov), spuhler@csc.kth.se (Jeannette Hiromi Spühler)
}

Unicorn consists of a small collection of finite element solver implementations for continuum mechanics models, to the most part based on general code with only a minimum of code dedicated to the particular model.

Simulation of turbulent flow is based on the General Galerkin $(\mathrm{G} 2)^{5}$ framework, where the effect of unresolved scales of turbulence is modeled by numerical dissipation from residual based stabilization, similar to an Implicit Large Eddy Simulation (LES) ${ }^{6}$. Similarly, unresolved shocks and discontinuities in compressible flow are treated by shock capturing stabilization.

Finite element methods have the benefit of a firm mathematical foundation which enables quantitative a posteriori error analysis, which forms the basis for adaptive methods where the computational mesh is modified to satisfy certain error tolerances guided by a posteriori error indicators. Fluid-structure interaction and deforming domains are treated by moving mesh algorithms and Arbitrary Lagrangian-Eulerian (ALE) methods. Parallel efficiency is essential, where adaptive methods for unstructured meshes pose particular challenges, and we here point out key algorithms of our software implementation.

The outline of the paper is the following: first we recall the basic continuum mechanics models we target, including turbulent flow and fluid-structure interaction, we then present the basic finite element algorithms underlying adaptivity and moving meshes, with particular focus on distributed parallel algorithms. We conclude the paper by a number of applications and a discussion of future work. 


\section{Basic models}

\subsection{The Navier-Stokes equations}

We consider fluid enclosed in a fixed, open domain $\Omega$ in three-dimensional space $\mathbb{R}^{3}$ with boundary $\Gamma$ over a time interval $I=[0, \hat{t}]$ with initial time zero and final time $\hat{t}$.

We seek the density $\rho$, momentum $\boldsymbol{m}=\rho \boldsymbol{u}$, with $\boldsymbol{u}=$ $\left(u_{1}, u_{2}, u_{3}\right)$ the velocity, and the total energy $E$ as functions of $(\boldsymbol{x}, t) \in Q \equiv \Omega \times I$, where $\boldsymbol{x}=\left(x_{1}, x_{2}, x_{3}\right)$ denotes the coordinates in $\mathbb{R}^{3}$. The equations for $\hat{\boldsymbol{u}} \equiv(\rho, \boldsymbol{m}, E) \mathrm{read}$ :

$$
\begin{aligned}
\partial_{t} \rho+\nabla \cdot(\rho \boldsymbol{u}) & =0 \\
\partial_{t} \boldsymbol{m}+\nabla \cdot(\boldsymbol{m} \otimes \boldsymbol{u}+\mathbb{I} p) & =\boldsymbol{g}+\nabla \cdot(2 \mu \varepsilon(\boldsymbol{u})+\lambda(\nabla \cdot \boldsymbol{u}) \mathbb{I}) \\
\partial_{t} E+\nabla \cdot(E \boldsymbol{u}+p \boldsymbol{u}) & =\nabla \cdot((2 \mu \varepsilon(\boldsymbol{u})+\lambda(\nabla \cdot \boldsymbol{u})) \cdot \boldsymbol{u}+\kappa \nabla T) \\
\hat{\boldsymbol{u}}(\cdot, 0) & =\hat{\boldsymbol{u}}^{0}
\end{aligned}
$$

where $p=p(\boldsymbol{x}, t)$ is the pressure of the fluid, $\otimes$ denotes the tensor product, $\mathbb{I}$ denotes the identity matrix in $\mathbb{R}^{3}, \partial_{t}=\partial / \partial t$, $\boldsymbol{g}=\left(\boldsymbol{g}_{1}, \boldsymbol{g}_{2}, \boldsymbol{g}_{3}\right)$ is a given volume force (e.g. gravity) acting on the fluid, $\hat{\boldsymbol{u}}^{0}=\hat{\boldsymbol{u}}^{0}(\boldsymbol{x})$ represents initial conditions,

$$
\varepsilon(\boldsymbol{u})=\frac{1}{2}\left(\nabla \boldsymbol{u}+\nabla \boldsymbol{u}^{T}\right),
$$

is the strain rate tensor, and $\kappa \geq 0$ the thermal conduction parameter. The viscosity parameters are assumed to satisfy conditions $\mu>0, \lambda+2 \mu>0$. For simplicity we sometimes use the approximation $\lambda=0$.

Further, the total energy $E=k+e$, where $k=\rho|\boldsymbol{u}|^{2} / 2$ is the kinetic energy, with $|\boldsymbol{u}|^{2} \equiv \boldsymbol{u}_{1}^{2}+\boldsymbol{u}_{2}^{2}+\boldsymbol{u}_{3}^{2}$, and $e=\rho T$ is the internal energy with $T$ the temperature scaled so that $c_{v}=1$, where $c_{v}$ is the heat capacity under constant volume.

For very high Reynolds numbers we may approximate the Navier-Stokes equations by inviscid flow, where the viscosity coefficients and thermal conductivity are zero, resulting in the Euler equations:

$$
\begin{aligned}
& \partial_{t} \rho+\nabla \cdot(\rho \boldsymbol{u})=0 \quad \text { in } Q, \\
& \partial_{t} \boldsymbol{m}+\nabla \cdot(\boldsymbol{m} \otimes \boldsymbol{u}+\mathbb{I} p)=\boldsymbol{g} \quad \text { in } Q \text {, } \\
& \partial_{t} E+\nabla \cdot(E \boldsymbol{u}+p \boldsymbol{u})=0 \quad \text { in } Q \text {, } \\
& \hat{\boldsymbol{u}}(\cdot, 0)=\hat{\boldsymbol{u}}^{0} \quad \text { in } \Omega
\end{aligned}
$$

The number of unknowns including the pressure is six but there are only five equations in (1)-(2); for a perfect gas, we close the system with the following state equation:

$$
p=(\gamma-1) e=(\gamma-1) \rho T=(\gamma-1)\left(E-\rho|\boldsymbol{u}|^{2} / 2\right),
$$

expressing the pressure $p$ as a function of density $\rho$ and temperature $T$, where $\gamma=c_{p}$ is the adiabatic index with $c_{p}$ the heat capacity under constant pressure, and $(\gamma-1)$ is the gas constant.

For a perfect gas, the speed of sound $c$ is given by $c^{2}=\gamma(\gamma-$ 1)T, and the Mach number is defined as $M=|\boldsymbol{u}| / c$, with $\boldsymbol{u}$ the velocity of the gas.

\subsection{Incompressible flow}

For low Mach numbers one may use the approximation of incompressible flow, corresponding to a divergence free condition on the velocity. The density may be variable, or for small density variations be approximated as constant $\rho_{0}$, leaving only the momentum equation and the divergence free condition. We then have the following equations: find $\hat{\boldsymbol{u}} \equiv(\boldsymbol{u}, p)$ such that:

$$
\begin{aligned}
\partial_{t} \boldsymbol{u}+(\boldsymbol{u} \cdot \nabla) \boldsymbol{u}+\nabla p & =\boldsymbol{g}+2 v \nabla \cdot \varepsilon(\boldsymbol{u}) & & \text { in } Q, \\
\nabla \cdot \boldsymbol{u} & =0 & & \text { in } Q, \\
\hat{\boldsymbol{u}}(\cdot, 0) & =\hat{\boldsymbol{u}}^{0} & & \text { in } \Omega .
\end{aligned}
$$

with $v=\mu / \rho_{0}$ the kinematic viscosity.

\subsection{Turbulent flow}

Direct Numerical Simulation (DNS) of turbulent flow is not possible in the general cases that we target, of high Reynolds numbers and complex geometry. In a Large Eddy Simulation (LES) ${ }^{6}$ only the largest scales of the flow are resolved, leaving the smallest, unresolved turbulent scales to be taken into account only through a subgrid model. Similarly, shocks and discontinuities in compressible flow can be left unresolved to be modeled through shock capturing regularization, and the effect of turbulent boundary layers can be approximated by wall shear stress models ${ }^{7}$.

Our approach to simulation of turbulent flow is based on the General Galerkin (G2) method, where numerical stabilization based on the residual of the equations models the effect of unresolved features in the flow, from turbulence to shocks ${ }^{5}$. The effect of unresolved turbulent boundary layers is modeled by a skin friction model for the wall shear stress .

\subsection{Unified continuum fluid-structure interaction}

For robustness we choose a monolithic approach to fluidstructure interaction (FSI), which we derive from the basic conservation laws. We here also seek a phase function $\theta$ and introduce the unified Cauchy stress $\sigma$ for all phases. The incompressible Unified Continuum fluid-structure model ${ }^{8}$ reads:

$$
\begin{array}{rlll}
\rho\left(\partial_{t} \boldsymbol{u}+(\boldsymbol{u} \cdot \nabla) \boldsymbol{u}\right)+\nabla \cdot \sigma & =\boldsymbol{g} & \text { in } Q, \\
\nabla \cdot \boldsymbol{u} & =0 & \text { in } Q, \\
\partial_{t} \theta+(\boldsymbol{u} \cdot \nabla) \theta & =0 & \text { in } Q, \\
\hat{\boldsymbol{u}}(\cdot, 0) & =\hat{\boldsymbol{u}}^{0} & \text { in } \Omega,
\end{array}
$$

where the phase function $\theta$ defines the solid and fluid domains by:

$$
\begin{aligned}
& \Omega_{s}(t)=\{x: x \in \Omega, \theta(x, t)=0\} \\
& \Omega_{f}(t)=\{x: x \in \Omega, \theta(x, t)=1\}
\end{aligned}
$$

For example, we can define a Newtonian fluid and an incompressible Neo-Hookean solid (here in stress rate form):

$$
\begin{aligned}
\sigma & =-\sigma_{D}+p I \\
\sigma_{D} & =\theta \sigma_{f}+(1-\theta) \sigma_{s} \\
\sigma_{f} & =2 \mu_{f} \varepsilon(\boldsymbol{u}) \\
\partial_{t} \sigma_{s} & =2 \mu_{s} \varepsilon(\boldsymbol{u})+\nabla \boldsymbol{u} \sigma_{s}+\sigma_{s} \nabla \boldsymbol{u}^{\top}
\end{aligned}
$$

where the subscript $s$ denotes solid and $f$ denotes fluid.

The FSI problem is thus treated as a multiphase flow problem, where a phase function $\theta$ identifies the solid and fluid, respectively. Typically we let the finite element mesh track the 
solid deformation and thus a piecewise constant phase function will not cut any elements in the mesh, but will stay with the solid throughout the computation, and thus no equation for the phase variable needs to be solved.

\section{Finite element approximation}

\subsection{General Galerkin finite element discretization}

Our computational approach is based on stabilized finite element methods, together with adjoint based adaptive algorithms, and residual based implicit turbulence modeling and shock capturing, for related work see e.g. ${ }^{9-11}$.

The General Galerkin (G2) method for high Reynolds number flow, including turbulent flow and shocks, takes the form of a standard Galerkin finite element discretization together with (i) least squares stabilization of the residual and (ii) residual based shock capturing.

With a G2 method, we define turbulent flow as the nonsmooth parts of the flow where the residual measured in $L_{2}$ norm increases as the mesh is refined, whereas in a negative $H^{-1}$-norm the residual decreases with mesh refinement ${ }^{12}$. That is, we characterize turbulence by a pointwise large residual which is small in average, corresponding to the equations being satisfied only in a mean value sense, which is sufficient to approximate mean value quantities of a turbulent flow field using $\mathrm{G} 2$.

We split the time interval $I$ into subintervals $I_{n}=\left(t_{n-1}, t_{n}\right)$, with associated space-time slabs $S_{n}=\Omega \times I_{n}$, over which we define space-time finite element spaces, based on a spatial finite element space $W^{n}$ over a spatial mesh $\mathcal{T}_{n}{ }^{5}$.

In a $\mathrm{cG}(1) \mathrm{cG}(1)$ method $^{13,14}$ we seek an approximate solution $\hat{U}=(U, P)$ which is continuous piecewise linear in space and time. With $W^{n}$ a standard finite element space of continuous piecewise linear functions, and $W_{0}^{n}$ the functions in $W^{n}$ which are zero on the boundary $\Gamma$, the $\mathrm{cG}(1) \mathrm{cG}(1)$ method for constant density incompressible flow with homogeneous Dirichlet boundary conditions for the velocity takes the form: for $n=1, \ldots, N$, find $\left(U^{n}, P^{n}\right) \equiv\left(U\left(t_{n}\right), P\left(t_{n}\right)\right)$ with $U^{n} \in V_{0}^{n} \equiv$ $\left[W_{0}^{n}\right]^{3}$ and $P^{n} \in W^{n}$, such that

$$
\begin{aligned}
& \left(\left(U^{n}-U^{n-1}\right) k_{n}^{-1}+\left(\bar{U}^{n} \cdot \nabla\right) \bar{U}^{n}, v\right)+\left(2 v \epsilon\left(\bar{U}^{n}\right), \epsilon(v)\right) \\
& -\left(P^{n}, \nabla \cdot v\right)+\left(\nabla \cdot \bar{U}^{n}, q\right) \\
& +S D_{\delta}^{n}\left(\bar{U}^{n}, P^{n} ; v, q\right)=(f, v), \forall \hat{v}=(v, q) \in V_{0}^{n} \times W^{n}
\end{aligned}
$$

where $\bar{U}^{n}=1 / 2\left(U^{n}+U^{n-1}\right)$ is piecewise constant in time over $I_{n}$, with the stabilizing term

$$
\begin{aligned}
S D_{\delta}^{n}( & \left.\bar{U}^{n}, P^{n} ; v, q\right) \equiv \\
\quad & \left(\delta_{1}\left(\bar{U}^{n} \cdot \nabla \bar{U}^{n}+\nabla P^{n}-f\right), \bar{U}^{n} \cdot \nabla v+\nabla q\right) \\
& +\left(\delta_{2} \nabla \cdot \bar{U}^{n}, \nabla \cdot v\right),
\end{aligned}
$$

where we have dropped the shock capturing term, and where

$$
\begin{aligned}
(v, w) & =\sum_{K \in \mathcal{T}_{n}} \int_{K} v \cdot w d x, \\
(\epsilon(v), \epsilon(w)) & =\sum_{i, j=1}^{3}\left(\epsilon_{i j}(v), \epsilon_{i j}(w)\right),
\end{aligned}
$$

with the stabilization parameters

$$
\begin{aligned}
& \delta_{1}=\kappa_{1}\left(k_{n}^{-2}+\left|U^{n-1}\right|^{2} h_{n}^{-2}\right)^{-1 / 2} \\
& \delta_{2}=\kappa_{2} h_{n}
\end{aligned}
$$

where $\kappa_{1}$ and $\kappa_{2}$ are positive constants of unit size. For turbulent flow we choose a time step size

$$
k_{n} \sim \min _{x \in \Omega}\left(h_{n} /\left|U^{n-1}\right|\right) .
$$

We note that the least squares stabilization omits the time derivative in the residual, which is a consequence of the test functions being piecewise constant in time for a $\mathrm{cG}(1)$ discretization of time ${ }^{14}$.

\subsection{Fluid-structure interaction}

For variable density incompressible flow and fluid-structure interaction the method takes a similar form, although in the first case we include a cG(1)cG(1) discretization of the equation for conservation of mass, and in the second case we add an equation for the structure stress.

For the Unified Continuum FSI model, we introduce a piecewise constant solid stress term $S_{s}$, and the mesh motion adds an ALE mesh velocity $\beta_{h}$ to the convective velocity:

$$
\begin{aligned}
& \left(\rho\left(\left(U^{n}-U^{n-1}\right) k_{n}^{-1}+\left(\left(\bar{U}-\beta_{h}\right)^{n} \cdot \nabla\right) \bar{U}^{n}\right), v\right) \\
& +(1-\theta)\left(S_{s}, \nabla v\right)+\theta\left(2 \mu_{f} \epsilon\left(\bar{U}^{n}\right), \epsilon(v)\right) \\
& -\left(P^{n}, \nabla \cdot v\right)+\left(\nabla \cdot \bar{U}^{n}, q\right) \\
& +S D_{\delta}^{n}\left(\bar{U}^{n}, P^{n} ; v, q\right)=(f, v), \forall \hat{v}=(v, q) \in V_{0}^{n} \times W^{n}
\end{aligned}
$$

\subsection{Explicit time stepping for compressible flow}

For compressible flow we add shock capturing terms, in the form of artificial viscosity based on the residual, and we find that for many problems this shock capturing stabilization appears sufficient to stabilize the system, and also acts as implicit subgrid model for turbulent flow ${ }^{15-17}$.

We typically choose explicit time stepping for compressible flow, although an implicit space-time formulation similar to the incompressible model is also possible ${ }^{18}$. With explicit time stepping, we first discretize the equations in space: find $\hat{U}_{h} \equiv$ $\hat{U}_{h}(t) \equiv\left(\rho_{h}(t), m_{h}(t), E_{h}(t)\right)$ with $\hat{U}_{h}(t) \in V_{h} \equiv W_{h} \times W_{h}^{3} \times W_{h}$, such that

$$
\left(\partial_{t} \hat{U}_{h}, \hat{v}_{h}\right)+\left(\nabla \cdot f\left(\hat{U}_{h}\right), \hat{v}_{h}\right)+\left(\nabla \cdot f_{v i s c}\left(\hat{U}_{h}\right), \hat{v}_{h}\right)=0,
$$

for all test functions $\hat{v}_{h}=\left(v_{h}^{\rho}, v_{h}^{m_{i}}, v_{h}^{E}\right) \in V_{h}$, where we have added residual based artificial viscosity to stabilize the system.

The resulting system of ordinary differential equations (5) is then solved by for example an explicit 3rd order Runge-Kutta method. We denote by $\hat{U}_{n}$ the solution at the discrete time steps $t_{n}$, that is $\hat{U}_{n} \equiv \hat{U}_{h}\left(t_{n}\right)$.

We define $U_{n}, P_{n}$ and $T_{n}$ to be finite element functions in $W_{h}^{3}$, $W_{h}$ and $W_{h}$ respectively, which are defined by their nodal values as

$$
\begin{aligned}
& U_{n}\left(N_{i}\right)=m_{n}\left(N_{i}\right) / \rho_{n}\left(N_{i}\right), \\
& P_{n}\left(N_{i}\right)=(\gamma-1) \rho_{n}\left(N_{i}\right) T_{n}\left(N_{i}\right), \\
& T_{n}\left(N_{i}\right)=E_{n}\left(N_{i}\right) / \rho_{n}\left(N_{i}\right)-\left|U_{n}\left(N_{i}\right)\right|^{2} / 2
\end{aligned}
$$


for all vertices $N_{i}$ in the mesh $\mathcal{T}_{n}$.

The fluxes are given as:

$$
f\left(\hat{U}_{n}\right)=\left(\begin{array}{r}
\rho U_{n} \\
m_{n} \otimes U_{n}+\mathbb{I} P_{n} \\
(E+P) U_{n}
\end{array}\right), f_{v i s c}\left(\hat{U}_{n}\right)=\left(\begin{array}{r}
-v_{n} \nabla \rho_{n} \\
-\mu_{n} \varepsilon\left(U_{n}\right) \\
-\mu_{n} \varepsilon\left(U_{n}\right) U_{n}-\kappa_{n} \nabla T_{n}
\end{array}\right)
$$

where the strain rate tensor is defined as above.

The dynamic viscosity $\mu_{n} \equiv \mu_{h}\left(t_{n}\right)$ is computed as follows: Let $\Delta t$ be a constant time-step and $\hat{U}_{n}, \hat{U}_{n-1}, \hat{U}_{n-2}$ are solutions at times $t_{n}, t_{n-1}, t_{n-2}$ respectively, where $\hat{U}_{n}$ is continuous piecewise linear in space.

Then, for each element $K$ we compute the residuals

$$
\begin{aligned}
& R_{\rho}\left(\hat{U}_{n}\right)=\frac{1}{2 \Delta t}\left(3 \rho_{n}-4 \rho_{n-1}+\rho_{n-2}\right)+\nabla \cdot\left(\rho_{n} U_{n}\right) \\
& R_{m}\left(\hat{U}_{n}\right)=\frac{1}{2 \Delta t}\left(3 m_{n}-4 m_{n-1}+m_{n-2}\right)+\nabla \cdot\left(m_{n} \otimes U_{n}+\mathbb{I} p_{n}\right), \\
& R_{E}\left(\hat{U}_{n}\right)=\frac{1}{2 \Delta t}\left(3 E_{n}-4 E_{n-1}+E_{n-2}\right)+\nabla \cdot\left(E_{n} U_{n}+p_{n} U_{n}\right) .
\end{aligned}
$$

We then take the maximum of the absolute value of the residuals in each element to compute the following residual based artificial viscosity:

$$
\begin{aligned}
\mu_{1, K}=C_{1} h_{K}^{2}\left\|\rho_{n}-\bar{\rho}_{n}\right\|_{\infty, \Omega} \max ( & \frac{\left\|R_{\rho_{n}}\right\|_{\infty, K}}{\left\|\rho_{n}-\bar{\rho}_{n}\right\|_{\infty, \Omega}}, \\
& \left.\frac{\left\|R_{m_{i}}\right\|_{\infty, K}}{\left\|m_{n_{i}}-\bar{m}_{n_{i}}\right\|_{\infty, \Omega}}, \frac{\left\|R_{E}\right\|_{\infty, K}}{\left\|E_{n}-\bar{E}_{n}\right\|_{\infty, \Omega}}\right)
\end{aligned}
$$

where $\left\|\rho_{n}-\bar{\rho}_{n}\right\|_{\infty, \Omega},\left\|m_{n_{i}}-\bar{m}_{n_{i}}\right\|_{\infty, \Omega},\left\|E_{n}-\bar{E}_{n}\right\|_{\infty, \Omega}$ are normalization terms, with $\bar{\rho}_{n}, \bar{m}_{n_{i}}, \bar{E}$ denoting space averaged values of the density, momentum and energy.

As in ${ }^{19,20}$ we compute the maximum dynamic viscosity, $\mu_{\max }$ as

$$
\mu_{\max , K}=C_{2} h_{K}\left\|\rho_{n}\right\|_{\infty, K}\left|\left\|u_{n} \mid+\sqrt{\gamma T_{n}}\right\|_{\infty, K}\right.
$$

and

$$
\left.\mu_{n}\right|_{K}=\min \left(\mu_{\max , K}, \mu_{1, K}\right),\left.\kappa_{n}\right|_{K}=\frac{\mathcal{P}}{\gamma-1} \mu_{n},\left.v_{n}\right|_{K}=\frac{\mathcal{P}}{\left\|\rho_{n}\right\|_{\infty, K}} \mu_{n}
$$

where $\mathcal{P} \approx 0.1$ is an artificial Prandtl number, $C_{1}=1$ usually, and $C_{2} \approx 0.25-0.5$.

\subsection{Boundary conditions}

Inflow boundary conditions are implemented in strong form through the finite element spaces, whereas outflow boundary conditions for incompressible flow are implemented in weak form as natural stress boundary conditions.

For compressible flow particular care has to be taken, where the appropriate boundary conditions depend on the Mach number, and are implemented thought the use of characteristics, see for example ${ }^{21}$. The characteristic boundary conditions are applied at inlet and outlet. Characteristic variables of the onedimensional Euler equations are found locally in each boundary element, which are then modified according to the incoming waves to approximate non-reflecting boundary conditions.
For the supersonic case all characteristics enter the computational domain at the inflow, whereas they leave the domain at the outflow. In this case Dirichlet boundary conditions are imposed for all variables at the inlet, but nothing is imposed at the outflow. For subsonic outflow, one boundary condition, typically for pressure, is imposed.

In the case of viscid laminar flow we use no slip boundary conditions at solid walls, with the fluid velocity given by the velocity of the solid wall. Turbulent boundary layers are modeled as a slip boundary condition, where the fluid velocity normal to the wall is given by the normal velocity of the solid wall, together with a skin friction boundary condition in the following form:

$$
\begin{aligned}
& u \cdot n=0, \\
& u \cdot \tau_{k}+\beta^{-1} n^{T} \sigma \tau_{k}=0, \quad k=1,2,
\end{aligned}
$$

for $(x, t) \in \Gamma_{\text {solid }} \times I$, with $n=n(x)$ an outward unit normal vector, and $\tau_{k}=\tau_{k}(x)$ orthogonal unit tangent vectors of the solid boundary $\Gamma_{\text {solid }}$. Here $\beta$ can be chosen as a constant parameter, or as a function of space and time, similar to simple wall shear stress models ${ }^{7,22}$ For very high Reynolds numbers we find that $\beta=0$ is a good approximation for small skin friction stress, which we have validated for a number of benchmark problems ${ }^{23,24}$

We implement the slip boundary condition in strong form, through a local projection of the velocity in normal and tangent directions, see ${ }^{15}$ for details. The tangential friction is imposed in weak form through the variational formulation.

\subsection{Moving mesh algorithms}

To handle deforming domains we employ an Arbitrary Lagrangian Eulerian (ALE) form of G2, which corresponds to a modification of the non-linear transport term by taking the mesh velocity into account. For the fluid mesh in a deforming domain, or when using r-adaptivity, we use either (i) mesh smoothing where the mesh connectivity is kept, or (ii) mesh transformations where the connectivity is changed.

Our preferred approaches are:

Laplacian/linear elastic mesh smoothing where the mesh velocity $\beta_{h}$ is given as a Dirichlet boundary condition, and a Laplacian or linear elastic boundary value problem is solved for the mesh velocity in the interior ${ }^{25}$. A basic variant smoothes the vertex coordinates instead by computing the average of the surrounding vertices.

Elastic mesh quality optimization where we use a formulation of time-dependent non-linear elasticity based on the deformation gradient $F$. By setting the initial condition $F_{0}=\bar{F}$, where $\bar{F}$ is the deformation gradient with regard to a scaled equilateral reference element, we formulate the problem of trying to find an optimal element quality as an elastic $\mathrm{PDE}^{8}$.

Topological mesh adaptation where we use topological mesh operations such as: refinement, coarsening and swapping 
to satisfy an element size and quality criterion. We use the MAdLib ${ }^{26}$ library for this purpose.

\subsection{A posteriori error estimation}

We base our adaptive algorithms on a posteriori estimation of the error in chosen output functionals of the solutions, such as forces, stresses, fluxes or local mean values. We follow the general framework for a posteriori error estimation based on the solution of associated dual problems, developed over the last 15 years. This field has many important contributors, for which we refer to the review papers ${ }^{27-29}$ and the references therein. Unicorn is based on the extension of the framework to turbulent flow $^{30-33}$ and to fluid-structure interaction ${ }^{34}$.

For $\hat{u}$ a weak solution of the equations, $\hat{U}$ a finite element approximation, and $\hat{\varphi}$ the solution of an associated dual problem, we derive the following a posteriori error estimate for a target functional $M(\hat{u})=((\hat{u}, \hat{\psi}))$, with $((\cdot, \cdot))$ the inner product over the space-time domain and $\hat{\psi}$ a weight function:

$$
|M(\hat{u})-M(\hat{U})| \leq \sum_{K \in \mathcal{T}_{n}} \mathcal{E}_{K}
$$

with error indicators of the form

$$
\begin{aligned}
\mathcal{E}_{K} \equiv \sum_{n=1}^{N}[ & \int_{I_{n}} \int_{K} \sum_{i}\left|R_{i}(\hat{U})\right|_{K} \cdot \omega_{i} d x d t \\
& \left.+\int_{I_{n}}\left|S D_{\delta}^{n}(\hat{U} ; \hat{\varphi})_{K}\right| d t\right]
\end{aligned}
$$

for each element $K$ in the mesh $\mathcal{T}_{n}$, with $R_{i}(\hat{U})$ residuals of the equations, and $S D_{\delta}^{n}(\cdot ; \cdot)_{K}$ is the stabilization over element $K$, and where the stability weights $\omega_{i}$ are given as derivatives of the dual solution $\hat{\varphi}$ multiplied by the local mesh size, and the dot denotes the scalar product in $\mathbb{R}^{3}$.

The error estimate thus naturally decouples into one standard Galerkin discretization error contribution, and one modeling error contribution from stabilization (representing also turbulence and shock capturing).

A posteriori analysis of fluid-structure interaction is challenging, but the Unified Continuum formulation, which effectively reduces FSI to a multiphase flow problem, enables the natural extension to FSI. For more details on a posteriori error estimation in Unicorn, including fluid-structure interaction, see $^{5,17,34}$.

\subsection{Adaptive algorithm}

In practice, the dual solution is approximated by a similar finite element method as we use for the primal problem, linearized at the primal solution. Based on the a posteriori error estimate we can then form adaptive algorithms for how to construct finite element meshes optimized to approximate the functional $M(\hat{u})$.

Starting from an initial coarse mesh $\mathcal{T}^{0}$, one simple such algorithm implemented in Unicorn takes the form: let $k=0$ then do

Algorithm 1. Adaptive mesh refinement
1. For the mesh $\mathcal{T}^{k}$ : compute the primal problem and the dual problem.

2. If $\sum_{K \in \mathcal{T}^{k}} \mathcal{E}_{K}<T O L$ then stop, else:

3. Mark some chosen percentage of the elements with highest $\mathcal{E}_{K}$ for refinement.

4. Generate the refined mesh $\mathcal{T}^{k+1}$, set $k=k+1$, and goto 1 .

\section{Parallelization strategy}

The parallelization is based on a fully distributed mesh approach, where everything from preprocessing, assembly of linear systems and postprocessing is performed in parallel, without representing the entire problem or any pre-/postprocessing step on one processing element (PE).

Inital data distribution is defined by the graph partitioning of the corresponding dual graph of the mesh. Each PE is assigned a set of whole elements and the vertex overlap between PEs is represented as ghosted entities.

\subsection{Parallel assembly}

The assembling of the stiffness matrix is performed in a straightforward fashion. Each PE computes the local stiffness matrix of the local elements and add them to the global matrix. Since we assign whole elements to each PE, we can minimize data dependency during assembly. Furthermore, we renumber all the degrees of freedom such that a minimal amount of communication is required when modifying entries in the sparse matrix.

\subsection{Solution of discrete system}

The FEM discretization in (4) generates a non-linear algebraic equation system to be solved for each time step. We solve this by iterating between the velocity and pressure equations by a Picard or quasi-Newton iteration ${ }^{8}$.

Each iteration in turn generates a linear system to be solved. We use simple Krylov solvers and preconditioners which scale well to many PEs, typically BiCGSTAB with a blockJacobi preconditioner, where each block is solved with ILU(0). DOLFIN uses the PETSc library as a parallel linear algebra back-end, which provides the linear algebra solvers.

For the case of explicit time-stepping for compressible flow we only need to solve one linear system per time step, which we similarly do with a simple Krylov solver.

\subsection{Mesh refinement}

Local mesh refinement is based around a parallelization of the well known recursive longest edge bisection method ${ }^{35}$. The parallelization split up the refinement into two phases. First a local serial refinement phase bisects all elements marked for refinement on each $\mathrm{PE}$ (concurrently) leaving several hanging nodes on the shared interface between PEs. The second phase propagates these hanging nodes onto adjacent PEs.

The algorithm iterates between local refinement and global propagation until all PEs are free of hanging nodes. The recursive nature of the longest edge bisection methods has the unpleasant feature that it can start bisecting elements in one 
PE, then propagate onto another PE and suddenly after several iterations between local refinement and global propagation, returning to the initial PE. Hence, in order to detect termination, we have to check for termination after each propagation phase. For an efficent implementation, one has to detect when all PEs are idling at the same time.

Our implementetaion uses a fully distributed termination detection scheme, which includes termination detection in the global propagation step by using recusive doubling or hypercube exchange type communication patterns ${ }^{36}$. The algorithm sends all propagation information to all PEs, regardless if they are taking part in the refinement or not. This approach may lead to a higher memory footprint, but if elements are selected for refinement using error control, these will be rather localized. Hence, several PEs will be idling (without any elements to refine) and can then be used to route the propagation of information without any risk of running out of memory.

Compared to other distributed termination detection algorithms, such as Dijkstra's general distributed termination algorithm $^{37,38}$, our implementation does not have a central point of control, hence no bottlenecks, less message contention, and no problems with load imbalance.

\subsubsection{Dynamic load balancing}

In order to sustain good load balance across several adaptive iterations, dynamic load balancing is needed. DOLFIN is equipped with a scratch and remap type load balancer, based on the widely used PLUM scheme ${ }^{39}$, where the new partitions are assigned in an optimal way by solving the maximally weighted bipartite graph problem. We have improved the scheme such that it scales linearly to thousands of cores ${ }^{36,40}$.

Furthermore, we have extended the load balancer with an a priori workload estimation. With a dry run of the refinement algorithm, we add weights to a dual graph of the mesh, corresponding to the workload after refinement. To serialize the refinement algorithm we use the concept of longest edge propgapation paths (LEPP) ${ }^{35}$ in the workload estimation, neglecting the propagation step.

Finally, we repartition the unrefined mesh according to the weighted dual graph and redistribute the new partitions before the refinement. This will lead to less data movement and more load balance in the bisection step ${ }^{35,41}$

\subsection{Mesh smoothing}

By formulating the mesh smoothing method as a PDE, we are able to exploit the parallel framework for automated solution of differential equations from FEniCS, such that no manual parallelization of the smoothing algorithms are necessary. The Laplacian/linear elastic smoothing and the elastic mesh quality optimization method in Unicorn are all formulated as a PDE.

\subsection{Parallel performance}

Parallel scalability and efficiency is essential for simulating flow past complex geometries. Unicorn has proven to scale well for a number of applications both strongly and weakly. Here we first present strong scaling results to demonstrate Unicorn's ability to efficently solve problems on a large number of PEs.

\subsubsection{Strong scaling}

We have performed the strong scalability tests on the 1516 node Cray XE6 Lindgren at PDC/KTH, measuring the time required for solving a single time step in two different configurations. The first one is a flexible mixer plate in turbulent flow (see $\$ 6.5$ ); with a mesh of $340 \mathrm{k}$ vertices, the scaling is optimal up to ca. 192 cores. The second configuration involves the turbulent flow past a full car model (see $\S 6.1$ ); with a mesh of $33 \mathrm{M}$ vertices, the scaling is optimal up to ca. 5000 cores. In Figure 1 we have plotted the result against the number of cores.

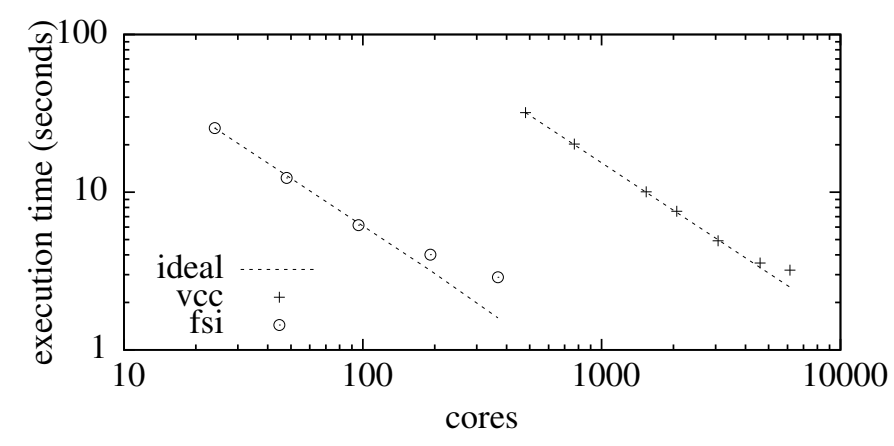

Figure 1: Strong scalability test on Lindgren. Here, $f$ si refers to the mixer plate configuration, whereas $v c c$ refers to the configuration of the flow past a full car model; the dashed lines display the ideal scaling for each configuration.

\subsubsection{Weak scaling}

The performance of an adaptive solver may deteriorate with the consecutive adaptive iterations, even if it performed well initally. This potential slow down can be caused, e.g., by increased communication and load imbalance. Weak scalabilty is therefore another interesting way of evaluating the solver's performance throughout several adaptive iterations.

We cannot freely scale the size our problems, as it is commonly done in standard weak scaling studies. Instead, we want to show that the computation time for a single element is constant over the entire iterative process. If so, the overhead introduced by the parallelization is minimal and the solver has a good weak scalability. Let $t_{\mathrm{s}}$ be the time required to compute a time step, $n_{\mathrm{e}}$ the number of elements in the mesh, and $n_{\mathrm{p}}$ the number of cores; we then define our weak scaling as:

$$
t_{\mathrm{c}}=\frac{t_{\mathrm{s}} n_{\mathrm{p}}}{n_{\mathrm{e}}}
$$

Here again, we have used the timings from two different applications, namely the flow past a full car model and the computation of aeroacoustic sources for a landing gear, see $\S 6.1$ and $\S 6.2$. In Figure 2 we see that our solver shows nearly optimal weak scaling, with an almost constant time per element across the entire adaptive process for both applications.

\section{Software implementation}

The algorithms and solvers described in this paper have been implemented in the unified continuum mechanics solver 


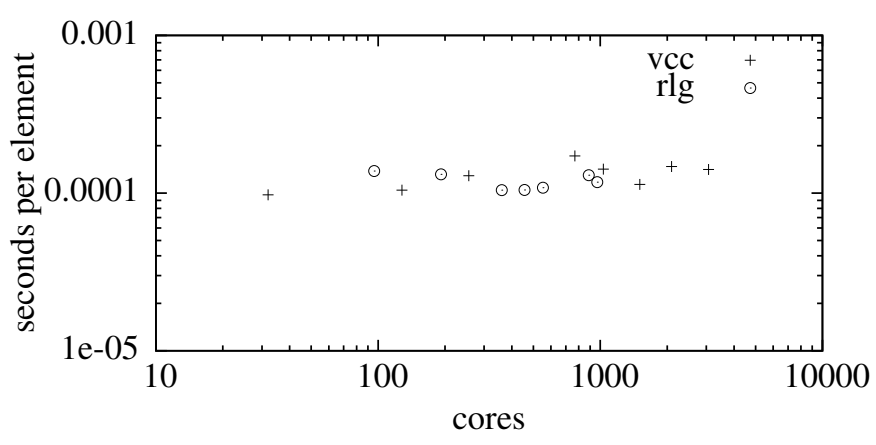

Figure 2: Weak scaling of the adaptive process for two different applications. Only one data point is plotted for each core count.

Unicorn and the high performance branch of the finite element problem solving environment DOLFIN. Both Unicorn and DOLFIN are written in $\mathrm{C}++$ and the implementation has proven to be portable across several different architectures, such as ordinary Unix/Linux workstations, IBM BlueGene/L and Cray XT6 and XE6 platforms.

The HPC branch of DOLFIN ${ }^{36}$ is optimized for distributed memory architectures using a hybrid MPI+OpenMP approach with efficient parallel I/O (MPI I/O). For mesh partitioning we use ParMETIS ${ }^{42}$ and for mesh adaptation we use MAdLib ${ }^{26}$. DOLFIN supports several parallel linear algebra packages; currently, nonetheless, we mostly rely on $\mathrm{PETSc}^{43}$.

\section{Applications}

We have outlined a computational framework for a large class of problems involving high Reynolds number flow, based on a G2 finite element discretization, a posteriori error control and adaptive algorithms, and strategies for parallel efficiency. The focus is on generality and simplicity, and in this section we illustrate the applicability of this framework for a set of challenging problems.

\subsection{Turbulent flow past a car}

Aerodynamic forces are of key importance in the design of a car, directly connected to fuel consumption. We have investigated the ability of Unicorn to accurately compute the forces on a full car model called Volvo Research into Automotive Knowledge (VRAK), for which there exists wind tunnel data.

Starting from an initial coarse tetrahedral mesh of 58k vertices and 280k elements, we have adaptively refined with respect to drag to obtain a mesh with $4.5 \mathrm{M}$ vertices and $24 \mathrm{M}$ tetrahedrons. The aerodynamic drag force converged to the available reference value and a total of thirteen adaptive iterations were performed. Figure 3 shows a snapshot from two different adaptive iterations. We may see that adaptivity mainly increases the mesh resolution where needed, typically in turbulent wakes, leaving larger elements in the free stream region, away from the car.

This challenging geometry together with the ability to sustain a large number of adaptive iterations demonstrate that Unicorn

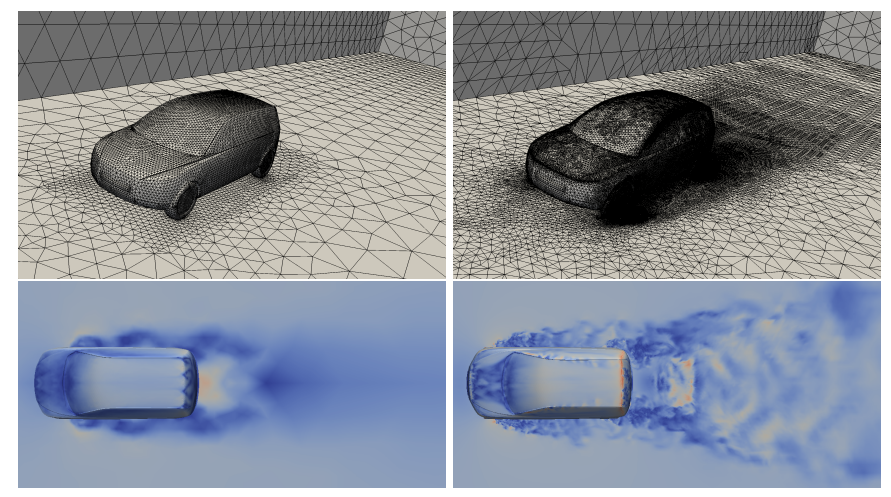

Figure 3: Mesh and velocity field at final time for two different iterations during the adaptive process.

is mature enough to be employed in the solution of realistic industrial problems. For a more detailed description of this simulation see ${ }^{44}$.

\subsection{Turbulent flow past a landing gear}

The Rudimentary Landing Gear (RLG) geometry was developed by Dr. Philippe Spalart and Dr. Kevin Mejia at Boeing Commercial Airplanes; it is a non-proprietary geometry specially designed for the Benchmark problems for Airframe Noise Computations I (BANC-I) workshop at the 16th AIAA/CEAS Aeroacoustics Conference in Stockholm, 2010. One of the main goals of this workshop was to assess available computational methods for the computation of aeroacoustic sources by comparing simulation results with experimental work. The results of our contribution ${ }^{24}$ included aerodynamic forces, $r m s$ of surface pressure and average pressure coefficient distributions, and they all were within acceptable ranges when compared with the results of other groups ${ }^{45}$.

Following the BANC-I workshop, we have used the RLG configuration to develop a new mesh refinement strategy ${ }^{46}$ that we believe is more suitable for computational aeroacoustics (CAA): instead of using the aerodynamic drag as the refinement target, we have chosen to employ a simplified form of Lighthill's tensor in our a posteriori error estimates. Lighthill's tensor is defined as $T \approx \rho u u^{T}$ and it is the source term in Lighthill's wave equation ${ }^{47}$, which can be used to predict sound propagation to the far field in incompressible, turbulent flows. Using this refinement strategy, for a similar mesh size, we were able to obtain a better resolution of the RLG's turbulent wake than with refinement based on drag. Thus, in this way, we expect to compute the aeroacoustic sources more accurately to a lower cost. To illustrate this result, Figure 4 shows instantaneous isosurfaces of vorticity using the Q-criterion. It is possible to see the difference between the two refinement cases: the Lighthill case resolves the turbulent scales in a larger volume of the wake. The two meshes used to obtain the results displayed in the figure contain 4.9M ("Lighthill refinement") and 5.0M ("drag refinement") elements and the computations were performed using 888 cores in the computer Lindgren at PDC, KTH. 

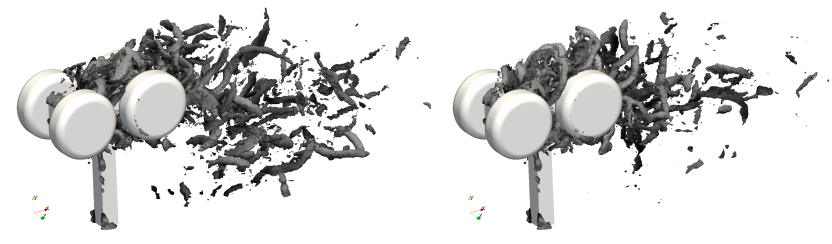

Figure 4: Isosurfaces of vorticity using the Q-criterion (left, Lighthill; right, drag). Both figures have been generated with meshes of equivalent sizes, ca. $5 \mathrm{M}$ elements.

\subsection{Ocean modeling}

Variable density, incompressible flow, together with a Coriolis force, is used as a simple ocean model in Unicorn to study basic problems of geoscience. We add the Coriolis term $(\boldsymbol{f} \times \boldsymbol{u})$ to the incompressible Navier-Stokes equations (3), where $\boldsymbol{f}=$ $f_{0} \hat{z}$. Here, $\hat{z}$ is the unit vector pointing in the z-direction and the Coriolis frequency is approximated by $f_{0}=2 \omega \sin \left(\vartheta_{0}\right)$, with $\omega$ the angular frequency of the planetary rotation and $\vartheta_{0}$ the latitude. The density of the atmosphere (or ocean) can be treated as incompressible and varies in the vertical direction. In order to solve for the density, we add to (3) the equation that implies the incompressibility condition. The equations now become:

$$
\begin{array}{rlll}
\rho\left(\partial_{t} \boldsymbol{u}+(\boldsymbol{u} \cdot \nabla) \boldsymbol{u}+(\boldsymbol{f} \times \boldsymbol{u})-\boldsymbol{g}\right) & & \\
+\nabla p-2 v \nabla \cdot \varepsilon(\boldsymbol{u}) & = & 0 & \text { in } Q, \\
\nabla \cdot \boldsymbol{u} & =0 & \text { in } Q, \\
\partial_{t} \rho+(\boldsymbol{u} \cdot \nabla) \rho & = & 0 & \text { in } Q \\
\hat{\boldsymbol{u}}(\cdot, 0) & = & \hat{\boldsymbol{u}}^{0} & \text { in } \Omega .
\end{array}
$$

As an example, we have studied the flow past a subsurface sea mountain. This is an interesting benchmark, since both numerical and theoretical works are available ${ }^{48}$, which allows us to evaluate our implemented model.
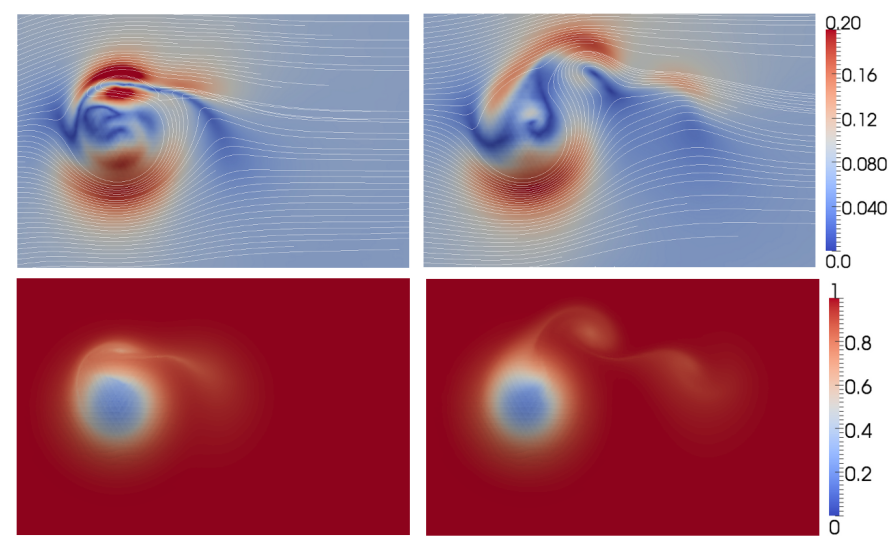

Figure 5: Velocity magnitude with streamlines (top) and density (bottom) for the flow past a sea mountain. (left, $\mathrm{T}=4$ days; right, $\mathrm{T}=24$ days ).

In Figure 5 we show the velocity magnitude as well as the density after 4 days and after 24 days for the flow past the sea mountain in 3D. The values are shown at the sea floor. Here, a Rossby number was $R o=\frac{U}{L f_{0}}=0.1$. Initially, the density field is linear in the $\mathrm{z}$-direction and it increases from $\rho=0$ at the surface to $\rho=1$ on the sea floor. A computation with $2.7 \mathrm{M}$ vertices and $13.5 \mathrm{M}$ tetrahedrons without adaptive mesh refinement was performed in parallel on 480 processors. It can be seen that a cyclonic eddy is shed from the sea mountain.

\subsection{Human heart model}

As an example of a problem with a deforming domain, we present our work on a model for blood flow in the left ventricle (LV) of the human heart.

The geometry is based on ultrasound measurements of the position of the inner wall of the LV at different time points during the cardiac cycle. We build a three dimensional mesh of tethrahedrons at the initial time and use a mesh smoothing algorithm to deform the mesh so that it fits the dynamic surface geometry. Finally, an adaptive ALE space-time finite element solver based on continuous piecewise linear elements in space and time together with streamline diffusion stabilization is used to simulate the blood flow by solving the incompressible Navier-Stokes equations. Pressure boundary conditions are prescribed to model inflow from the mitral valve and outflow through the aortic valve, see ${ }^{49}$ for more details.

Blood flow profiles are validated against patient specific measurements, with a good agreement. See Figure 6 for a visualization of the blood flow and the deforming mesh.
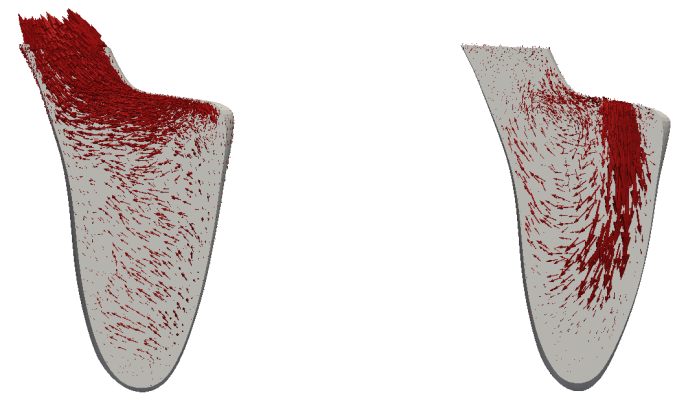

Figure 6: Velocity field during diastole (right) and systole (left).

\subsection{Fluid-structure interaction and turbulence}

An experimental configuration approximating an exhaust system with a flexible triangular steel plate in a circular duct flow has been studied in ${ }^{50}$. The Reynolds number is $2.55 \times 10^{5}$ at a Mach number of 0.12 . The flow induces a static deflection and oscillation of the plate. How this oscillation influences the aero-acoustical properties poses an interesting research question.

We set up the duct and flow conditions in Unicorn and introduce a flexible plate. Representative snapshots of the velocity and pressure together with the elastic plate are given in Figure 7.

The FSI solver algorithm in every time step consists of assembly and linear solves of momentum and pressure equations, assembly of the Cauchy stress and mesh motion including smoothing. We show the parallel performance of this solver in a strong scaling test in Figure 1. 

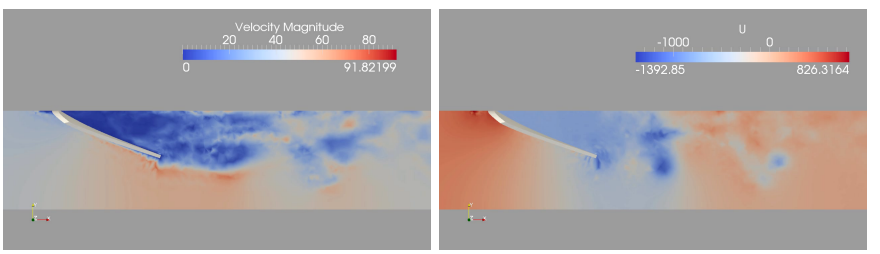

Figure 7: Snapshot of the velocity field and flexible mixer plate (left) and pressure field and plate (right).

\subsection{Turbulent compressible flow}

We have performed the numerical computation of high Reynolds number transonic compressible flow past a circular cylinder in $3 \mathrm{D}^{17}$. The Dirichlet boundary condition corresponding to Mach number 0.8 are imposed at the inflow. Non-reflecting boundary conditions are set at the outflow, and, for the remaining boundaries, slip boundary conditions are imposed.

The initial mesh consisted of $3.4 \mathrm{k}$ vertices and $15.5 \mathrm{k}$ elements. After each adaptive iteration, $5 \%$ of the elements with the highest error contributions are marked for refinement. The final mesh, which was obtained after 12 adaptive iterations, has $51.4 \mathrm{k}$ vertices and $265 \mathrm{k}$ elements, see Figure 8.

In Figure 8 we plot isosurfaces of the magnitude of the dual momentum, and the velocity, from the finest mesh. The primal solution shows attached shock-waves and a von-Karman vortex street. The dual problem is computed with the drag force of the cylinder as the target functional. The adaptive mesh refinement allows for an efficient computation of the drag force, where the results are consistent with experimental values ${ }^{17}$.

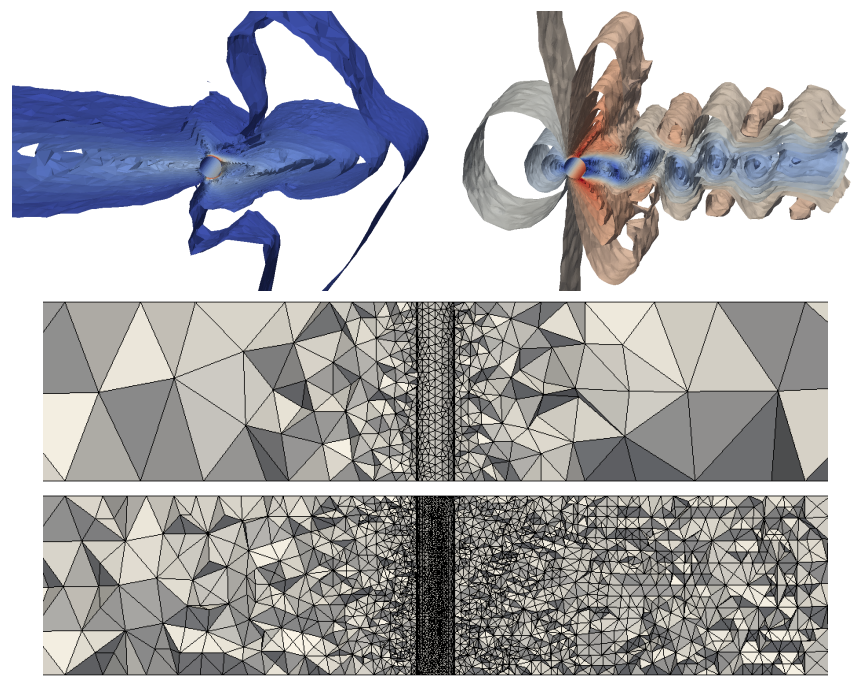

Figure 8: Transonic flow past a circular cylinder: the magnitude of the dual momentum $0 \leq\left|\varphi_{m}\right| \leq 6$ (upper-left) at $t=9.5$, the primal velocity $0 \leq|U| \leq$ 1.593 (upper-right) at $t=15.9265$. The initial and the final meshes are plotted in the lower part of the figure.

\section{Summary and future work}

A computational framework for adaptive finite element simulation of turbulent flow and fluid-structure interaction is pre- sented, including efficient parallel implementation. The computational tools are implemented open source as Unicorn and a high performance branch of DOLFIN, freely available as part of the FEniCS project ${ }^{4}$.

Future work includes further unification of the different solvers in Unicorn, and extensions of the basic methodology to a larger class of continuum mechanics problems.

\section{Acknowledgments}

The authors would like to acknowledge the financial support from the European Research Council, Swedish Foundation for Strategic Research, the Swedish Research Council, and the Swedish Energy Agency. The car geometry was generously made available to us by Volvo Car Corporation. The simulations were performed on resources provided by the Swedish National Infrastructure for Computing (SNIC) at High Performance Computing Center North (HPC2N), National Supercomputer Centre in Sweden (NSC) and PDC - Center for HighPerformance Computing.

\section{References}

[1] Logg A, Wells GN. DOLFIN: Automated finite element computing. ACM Trans Math Softw 2010;37(2):1-28.

[2] Logg A, Wells GN, Hake J, et al. DOLFIN: A C++/Python finite element library. 2011. http://launchpad.net/dolfin.

[3] Hoffman J, Jansson J, Nazarov M, Jansson N. Unicorn. 2011. http://launchpad.net/unicorn/hpc.

[4] FEniCS . FEniCS project. 2003. http://www.fenicsproject.org.

[5] Hoffman J, Johnson C. Computational Turbulent Incompressible Flow; vol. 4 of Applied Mathematics: Body and Soul. Springer; 2007.

[6] Sagaut P. Large Eddy Simulation for Incompressible Flows (3rd Ed.). Springer-Verlag, Berlin, Heidelberg, New York; 2005.

[7] Piomelli U, Balaras E. Wall-layer models for large-eddy simulation. Annu Rev Fluid Mech 2002;34:349-374.

[8] Hoffman J, Jansson J, Stöckli M. Unified continuum modeling of fluidstructure interaction. Math Mod Meth Appl S 2011;

[9] Bazilevs Y, Calo V, Cottrell J, Hughes T, Reali A, Scovazzi G. Variational multiscale residual-based turbulence modeling for large eddy simulation of incompressible flows. Comput Meth Appl Mech Eng 2007;197(14):173-201.

[10] Guasch O, Codina R. A heuristic argument for the sole use of numerical stabilization with no physical les modeling in the simulation of incompressible turbulent flows. Preprint Universitat Politecnica de Catalunya 2007; .

[11] Guermond JL, Pasquetti R, Popov B. From suitable weak solutions to entropy viscosity. J Scientific Comput 2011;49:35-50.

[12] Hoffman J, Johnson C. Blow up of incompressible euler equations. BIT 2008;48:285-307.

[13] Hansbo P. A crank-nicolson type space-time finite element method for computing on moving meshes. J Comput Phys 2000;159:274-289.

[14] Hoffman J, Jansson J, de Abreu RV. Adaptive modeling of turbulent flow with residual based turbulent kinetic energy dissipation. Comput Meth Appl Mech Eng 2011;200(37-40):2758 - 2767.

[15] Nazarov M. Adaptive algorithms and high order stabilization for finite element computation of turbulent compressible flow. Ph.D. thesis; KTH, Numerical Analysis, NA; 2011. QC 20110627.

[16] Nazarov M. Convergence of a residual based artificial viscosity finite element method. Tech. Rep. KTH-CTL-4015; Computational Technology Laboratory; 2011. http://www.publ.kth.se/trita/ctl-4/015/.

[17] Nazarov M, Hoffman J. Residual based artificial viscosity for simulation of turbulent compressible flow using adaptive finite element methods. Int J Numer Methods Fluids accepted;. 
[18] Nazarov M, Hoffman J. An adaptive finite element method for inviscid compressible flow. Int J Numer Methods Fluids 2010;64(188):11021128. doi:10.1002/fld.2335.

[19] Guermond JL, Pasquetti R. Entropy-based nonlinear viscosity for fourier approximations of conservation laws. C R Acad Sci 2008;Ser. I, 346:801806.

[20] Guermond JL, Pasquetti R, Popov B. Entropy viscosity method for nonlinear conservation laws. J Comput Phys 2011;230(2011):4248-4267.

[21] Löhner R. Applied CFD techniques: an introduction based on finite element methods. John Wiley \& Sons; 2001.

[22] Schumann U. Subgrid scale model for finite difference simulations of turbulent flows in plane channels and annuli. J Comput Phys 1975;18(4):376 $-404$.

[23] Hoffman J, Jansson N. A computational study of turbulent flow separation for a circular cylinder using skin friction boundary conditions. In: Quality and Reliability of Large-Eddy Simulations II; vol. 16 of ERCOFTAC Series. Springer Netherlands; 2011, p. 57-68.

[24] de Abreu RV, Jansson N, Hoffman J. Adaptive computation of aeroacoustic sources for rudimentary landing gear. In: proceedings for Benchmark problems for Airframe Noise Computations I, Stockholm. 2010,

[25] Löhner R, Yang C. Improved ALE mesh velocities for moving bodies. Commun Numer Meth En 1996;12(10):599-608.

[26] Compère G, Remacle J, Jansson J, Hoffman J. A mesh adaptation framework for dealing with large deforming meshes. Int J Numer Methods Eng 2010;82(7):843-867.

[27] Eriksson K, Estep D, Hansbo P, Johnson C. Introduction to adaptive methods for differential equations. Acta Numer 1995;4:105-158.

[28] Becker R, Rannacher R. A posteriori error estimation in finite element methods. Acta Numer 2001;10:1-103.

[29] Giles M, Süli E. Adjoint methods for pdes: a posteriori error analysis and postprocessing by duality. Acta Numer 2002;11:145-236.

[30] Hoffman J. Computation of mean drag for bluff body problems using adaptive dns/les. SIAM J Sci Comput 2005;27(1):184-207.

[31] Hoffman J, Johnson C. A new approach to computational turbulence modeling. Comput Methods Appl Mech Engrg 2006;195:2865-2880.

[32] Hoffman J. Adaptive simulation of the subcritical flow past a sphere. J Fluid Mech 2006;568:77-88.

[33] Hoffman J. Efficient computation of mean drag for the subcritical flow past a circular cylinder using general Galerkin G2. Int J Numer Meth Fl 2009;59(11):1241-1258.

[34] Degirmenci N, Hoffman J, Jansson J. An adaptive finite element method for unified continuum fluid-structure interaction. Tech. Rep. KTH-CTL-4020; Computational Technology Laboratory; 2011. http://www.publ.kth.se/trita/ctl-4/020/.

[35] Rivara M. New longest-edge algorithms for the refinement and/or improvement of unstructured triangulations. Int J Numer Meth Eng 1997;40(18):3313-3324.

[36] Jansson N. High performance adaptive finite element methods for turbulent fluid flow. Licentiate thesis; Royal Institute of Technology; School of Computer Science and Engineering; 2011. TRITA-CSC-A 2011:02.

[37] Dijkstra EW, Scholten CS. Termination detection for diffusing computations. Inform Process Lett 1980;11(1):1 - 4 .

[38] Bertsekas DP, Tsitsiklis JN. Parallel and Distributed Computation: Numerical Methods. Athena Scientific; 1997.

[39] Oliker L. PLUM parallel load balancing for unstructured adaptive meshes. Tech. Rep. RIACS-TR-98-01; RIACS; NASA Ames Research Center; 1998.

[40] Jansson N, Hoffman J, Jansson J. Performance of Dolfin and Unicorn on modern high-performance distributed memory architectures. Tech. Rep. KTH-CTL-4012; Computational Technology Laboratory; 2010. http://www.publ.kth.se/trita/ctl-4/012.

[41] Schloegel K, Karypis G, Kumar V, Biswas R, Oliker L. A Performance Study of Diffusive vs. Remapped Load-Balancing Schemes. In: 11th Intl. Conference on Parallel and Distributed Computing Systems. 1998,

[42] Schloegel K, Karypis G, Kumar V. ParMETIS, Parallel graph partitioning and sparse matrix ordering library. 2011.

[43] Balay S, Buschelman K, Gropp WD, Kaushik D, Knepley MG, McInnes LC, et al. PETSc Web page. 2009. Http://www.mcs.anl.gov/petsc.

[44] Jansson N, Hoffman J, Nazarov M. Adaptive Simulation of Turbulent Flow Past a Full Car Model. In: Proceedings of the 2011 ACM/IEEE International Conference for High Performance Computing, Networking,
Storage and Analysis. SC '11; 2011

[45] Spalart PR, Mejia K. Analysis of experimental and numerical studies of the rudimentary landing gear. In: Proceedings for the 49th AIAA Aerospace Sciences Meeting including the New Horizons Forum and Aerospace Exposition, Orlando, Florida. 2011,

[46] de Abreu RV, Jansson N, Hoffman J. Adaptive computation of aeroacoustic sources for a rudimentary landing gear using lighthill's analogy. In: Proceedings for the 17th AIAA/CEAS Aeroacoustics Conference (32nd AIAA Aeroacoustics Conference), Portland, Oregon. 2011,

[47] Lighthill MJ. On sound generated aerodynamically. Proc R Soc Lond A 1952;211:564-587.

[48] Ford R, Pain C, Piggott M, Goddard A, de Oliveira C, Umpleby A. A nonhydrostatic finite-element model for three-dimensional stratified oceanic flows. part ii: Model validation. Mon Weather Rev 2004;132(12):28322844.

[49] Aechtner M. Arbitrary Lagrangian-Eulerian Finite Element Modelling of the Human Heart. Master's thesis; Royal Institute of Technology; School of Computer Science and Engineering; 2009. TRITA-CSC-E 2009:022.

[50] Karlsson M, Holmberg A, Åbom M, Fallenius B, Fransson J. Experimental determination of the aero-acoustic properties of an in-duct flexible plate. In: Proceedings for 14th AIAA/CEAS Aeroacoustics Conference (29th AIAA Aeroacoustics Conference), Vancouver, British Columbia. 2008 , 